\title{
BMJ open Estimate of HIV prevalence and number of people living with HIV in India 2008-2009
}

\author{
Arvind Pandey, ${ }^{1}$ Damodar Sahu, ${ }^{1}$ Taoufi Bakkali, ${ }^{2}$ DCS Reddy, ${ }^{3}$ S Venkatesh, ${ }^{4}$ \\ Shashi Kant, ${ }^{5}$ M Bhattacharya, ${ }^{6}$ Yujwal Raj, ${ }^{4}$ Partha Haldar, ${ }^{3}$ Deepak Bhardwaj, ${ }^{1}$ \\ Nalini Chandra ${ }^{2}$
}

To cite: Pandey A, Sahu D, Bakkali T, et al. Estimate of HIV prevalence and number of people living with HIV in India 2008-2009. BMJ Open 2012;2:e000926.

doi:10.1136/bmjopen-2012000926

- Prepublication history for this paper are available online. To view these files please visit the journal online (http://dx.doi.org/10.1136/ bmjopen-2012-000926).

Received 9 February 2012 Accepted 14 August 2012

This final article is available for use under the terms of the Creative Commons Attribution Non-Commercial 2.0 Licence; see http://bmjopen.bmj.com

For numbered affiliations see end of article.

Correspondence to Dr Arvind Pandey; arvindp.nims@gmail.com

\section{ABSTRACT}

Objectives: To update the estimation of the adult HIV prevalence and number of people living with HIV (PLHIV) in India for the year 2008-2009 with the combination of improved data and methods.

Design: Based on HIV sentinel surveillance (HSS) data and a set of epidemiological assumptions, estimates of HIV prevalence and burden in India have been derived.

Setting: HSS sites spread over all the States of India. Participants: Secondary data from HSS sites which include attendees of antenatal clinics and sites under targeted interventions of high-risk groups, namely, female sex workers (FSW), intravenous drug users (IDU) and men having sex with men (MSM).

\section{Primary and secondary outcome measures:}

Estimates of adult HIV prevalence and PLHIV in India and its states.

Results: The adult HIV prevalence in India has declined to an estimated $0.31 \%(0.25-0.39 \%)$ in 2009 against $0.36 \%(0.29-0.45 \%)$ in 2006. Among the high prevalence states, the HIV prevalence has declined in Tamil Nadu to $0.33 \%$ in 2009 and other states show either a plateau or a slightly declining trend over the time period 2006-2009. There are states in the low prevalence states where the adult HIV prevalence has risen over the last 4 years. The estimated number of PLHIV in India is 2.4 million (1.93-3.04 million) in 2009. Of which, $39 \%$ are women, children under 15 years of age account for $4.4 \%$ of all infections, while people aged $15-49$ years account for $82.4 \%$ of all infections.

Conclusions: The estimated adult prevalence has declined in few states, a plateau or a slightly declining trend over the time. In future, efforts may be made to examine the implications of the emerging trend of the HIV prevalence on the recent infections in the study population.

\section{BACKGROUND}

Based on HIV sentinel surveillance (HSS) data and a set of epidemiological assumptions, estimates of HIV prevalence and

\section{ARTICLE SUMMARY}

Article focus

- Modelling exercise to estimate the adult prevalence and burden of HIV in India using the HIV Sentinel Surveillance (HSS) data.

Key messages

- Estimates indicate a slow-down trend in the epidemic with current adult HIV prevalence at $0.31 \%$ in 2009 .

- In 2009, 2.39 million people were estimated to be living with HIV with uncertainty bounds 1.93-3.04 millions.

- Men account for a greater proportion of the epidemic's burden vis-à-vis women at $61 \%$ and $39 \%$, respectively. The percentage distribution of HIV infection by age is estimated at $4 \%$ among children below the age of 15 years, $83 \%$ among adults and $13 \%$ among those aged over 50 years.

Strengths and limitations of this study

- Epidemiological assumptions used in modelling based on evidence on limited studies in other countries, not on Indian population.

burden in India have been derived every year since 1998. The process is consultative among the national and international experts in the field of biostatistics and epidemiology. Technical supports are received from UN organisations particularly experts from the WHO and UNAIDS (United Nations Programme on AIDS). Efforts are made to improve data both in terms of quality and representativeness as well as the methodology matching to epidemics. For instance, in 2006 with the expansion of sentinel surveillance to all districts, ${ }^{1}$ sero-survey as a part of third round of National Family Health Survey (NFHS-3) ${ }^{2}$ and the observation that there is a common practice of referral of HIV-positive/suspected cases to public hospitals and a preferential use of public 
hospitals by people in the lower socio-economic strata causing overestimation of the HIV burden in India, ${ }^{3} 4$ many of the assumptions are replaced with evidencebased information.

In fact, the NFHS-3 provided an opportunity to calibrate the surveillance data $^{5}{ }^{6}$ particularly the data from antenatal clinic (ANC) attendees used in the estimation process as a proxy to the general population. In addition, globally used method for similar epidemic, for example, WHO/UNAIDS workbook $^{7}$ along with Spectrum software ${ }^{8}$ was used in 2006 and 2007 rounds of estimation. The WHO/UNAIDS Workbook having assimilated the average prevalence for each risk group, namely, FSW, IDU, MSM and ANC fitted a logistic model to get the trend of the epidemic. The calibration factors derived in 2006 were used in the 2007 round of estimation in view of non-availability of community-based survey data to calibrate every year. ${ }^{9}$ Also, it forwarded a limitation of curve fitting over the average HIV prevalence in the population within the Workbook. The issue was deliberated in the UNAIDS Global Reference group on Estimates, Modelling and Projections and it was suggested to adopt the combination of Estimation and Projection Package (EPP) and Spectrum. ${ }^{10}$ The present paper aims to update the HIV estimation with the above combination. Specifically, it describes the data and methods used for the 2008 and 2009 HIV burden estimates for India and compares the resulting estimates with those of earlier years.

\section{MATERIAL AND METHOD}

$\mathrm{EPP}^{11}$ and Spectrum DemProj and AIDS Impact Model (AIM) modules ${ }^{12}$ were used for estimating prevalence and burden of HIV.

\section{Data}

The Working Group reviewed and used four sets of data available from various sources for the estimation process.

\section{Surveillance}

The following surveillance data available from 1998 to 2009 was used in EPP for producing state and national curves. These represent HIV prevalence data from HSS that was conducted among pregnant women attending antenatal clinics and the key population groups of female sex workers (FSW), men having sex with men (MSM) and injecting drug users (IDUs). Through the scale-up in number of HSS sites and increased focus on key population sites, a wider geographical and population group coverage was achieved leading to more accurate results. Table 1 reflects the scale-up in numbers of surveillance sites per year for the population groups that are used in the HIV estimates process.

Also, the working group used the estimated prevalence from the National Family and Health Survey (NFHS) conducted throughout the country with state-level representation in the high prevalence states. The adult HIV prevalence from the NFHS-3 was used for calibration of the fitted curves.

\section{Population sizes (the size of the higher risk and lower risk population)}

Second, for determining specific demographic parameters of key population groups, the size estimates for FSW, MSM and IDU provided under the NACP-III document ${ }^{13}$ was considered. This was updated with data from the 2009 mapping exercise conducted among key population in 2009 in specific states by National AIDS Control Organization (NACO) and State AIDS Control Societies (SACs). In states where the mapping exercise was not concluded, the higher-risk group population was estimated as a proportion of people with higher-risk behaviour as reported under the NACP-III document. ${ }^{14}$

In addition, data inputs used for determining the size of the general population at lower risk for HIV were number of births, number of deaths, adult population growth rate and population size for people aged over 15 years across and in 34 states/Union Territories from the vital registration system. ${ }^{15}$ National population estimates were obtained from population projection for India and states from the period 2001 to 2026. The size of the general population at lower risk for HIV were calculated as the total adult population minus the population size of the higher risk groups. The population size for people aged 15-49 years in 2009 has been derived through Demproj in the Spectrum Package. The data used in Demproj for calculating the population size included the Census population data of 1981, 1991 and 2001 and the Expert Group Population Estimates and Projections of India. ${ }^{16-19}$ The breakdown by sex for the IDU population assumes that $90 \%$ of the IDU population is male and $10 \%$ is female. ${ }^{13} 20$ 


\section{Programme coverage}

As antiretroviral therapy (ART) programme coverage influences the trend of HIV prevalence, the current ART coverage is extrapolated for the years beyond 2009which is consistent with the NACP-III planned target of 500000 by 2015-and distributed among all risk groups based on last year proportions in respective risk groups. ${ }^{21}$

\section{Methods}

The EPP estimates the trends over time of HIV prevalence by fitting an epidemiological model to the surveillance data provided by HSS systems. The basic principle underlying EPP is to develop epidemic curves separately for different subpopulations and then combine to produce a single epidemic curve estimating HIV prevalence at the national level. For adult HIV prevalence epidemic curve generated for each subpopulation group, initial guesses were made using four parameters, namely $\mathrm{t}_{0}$ (start year of the HIV epidemic), $r$ (the force of infection), $f_{0}$ (the initial fraction of the adult population at risk of infection used to determine the peak level of the epidemic curve, $\varphi$ (the behaviour adjustment parameter which determines how the proportion of new entrants in the adult population who are at risk of HIV infections changes over time). The 2009 version has also included the provision of ART, which increases survival of people living with HIV (PLHIV), hence increasing prevalence and impacting on the process of fitting an epidemiological model to the HIV epidemic. Data from the above sources were entered to EPP to produce curves of adult HIV prevalence among different population groups including ANC attendees - as proxy for general population-and key population groups.

The following two primary epidemiological assumptions were considered while analysing data under the 2009 version of EPP: (1) a specific criterion was considered for reassigning higher-risk groups to the general population category. Based on the second round of Behaviour Surveillance Survey conducted in $2006,{ }^{20}$ it was determined that IDU and MSM after a 15-year duration would be reassigned to the general population category whereas for FSW the timeframe for being reassigned to the general population was reduced to 8 years and (2) non-AIDS mortality was assumed to be higher by as much as $7 \%$ for injecting drug users vis-à-vis non-injecting drug users meant to account for the higher risk of mortality experienced by all IDU regardless of HIV status.

\section{Defining the characteristics of the epidemic}

The HIV epidemic in India, at national level, is concentrated among FSW, MSM and IDU. Among the 34 Indian States/Union Territories-the epidemic is defined as a concentrated non-IDU epidemic for all the states with the exception of Manipur and Nagaland where the epidemic is defined as a concentrated IDU epidemic.
Generating state-specific prevalence curves

The States/Union Territory-specific epidemic, for which first time States/Union Territory-specific prevalence curves was generated under the 2008-2009 HIV estimates. Using the Bayesian melding approach, 1000 iterations were used for fitting the initial guesses for ANC sites and 3000 iterations for higher-risk group sites. The bestfitting curves for all subpopulation categories were subsequently combined for producing state prevalence curves.

\section{Calibrating ANC prevalence curves in EPP model}

Estimates of HIV prevalence is primarily based on timesseries prevalence data among ANG attendees in HSS. Due to the difference in sero-prevalence between the ANC attendees and those from population-based surveys, the calibration of the prevalence curves based on the former is required. The key source of information used for calibrating HIV prevalence curves has the 2006 NFHS- $3^{2}$ where state-specific information on HIV prevalence is determined. The curve for ANC attendees was calibrated from NFHS-3 2005-2006 for the general population. In order to have an appropriate calibration of the HIV prevalence trends determined in Spectrum, the point values of prevalence determined from the NFHS-3 in 2006 as a reference for calibrating the prevalence trend for general population, initially determined through ANC.

In EPP, when a calibration factor is used, the overall curve determined on the basis of ANC HSS trend data which is scaled according to the calibration constant. Differences in the prevalence level are modelled on the probit scale. The probit scale is chosen in such a way that the differences between prevalence levels do not depend on the level itself. The calibration factor was derived for individual states in five high-prevalence states, that is, Andhra Pradesh, Karnataka, Maharashtra, Manipur and Tamil Nadu, based on calculations from NFHS-3, while for Nagaland the calibration factor was determined from a specific study undertaken by NACO. ${ }^{22}$ For the remainder of the moderate and lowprevalence states, the common constant calibration factor of 0.69 was derived from the NFHS-3 on the basis of the rest of the states (excluding aforesaid six high prevalence states) national comparison between general population prevalence and ANC prevalence.

\section{Estimation of PLHIV for all age groups using Spectrum}

In order to estimate the number of PLHIV and HIV prevalence for all ages, the projected adult HIV prevalence for each state was fed into Spectrum along with programme data on ART programme coverage, percentage of mothers and children given nevirapine prophylaxis and certain demographic and epidemiological parameters.

Spectrum under its AIM requires a number of inputs and parameters to process estimates and projections of HIV-related parameters that will allow fitting of the trend of epidemic as initially determined in EPP. 
The first input into Spectrum is the projection of HIV incidence determined in EPP. It is combined with the population projection and the other programme coverage indicators and parameters to determine the indicators related to the impact of the epidemic. The parameters included into Spectrum include the ART, Prevention of Parent to Child Transmission of HIV/AIDS (PPTCT) programme coverage data, age and sex distribution of prevalence. For each of the 34 States/Union Territories, adult and children ART treatment coveragefrom 2004 to 2009 and the projected coverage till 2015along with duration of breastfeeding were used in AIM. An estimated 300000 adults and 17000 children utilised ART as on December 2009. Approximately 13000 mothers had utilised PPTCT in India as on December 2009. Assumptions over other state-specific HIV characteristics included age and sex distribution of new infections, proportion of those newly infected progressing need for treatment by time since infection, proportion of adults in need of treatment, proportion of adults dying due to AIDS-related causes without treatment by time in need, annual mortality among children in need of treatment but not receiving treatment by age, annual survival of adults and children on ART, probability of transmission of HIV from mother-to-child, etc. ${ }^{12}{ }^{23}$ After finalising the input of all these parameters, Spectrum reprocessed the estimation and projection of the HIV epidemic.

\section{Uncertainty analysis}

The point estimates are associated with uncertainty due to the prevalence/incidence curve produced by EPP and the input assumptions that are based on studies from population samples in selected countries. For addressing this associated uncertainty, a special programme in Spectrum ${ }^{24}$ was used for producing uncertainty bounds around the usual point estimates for each indicator and for each year. A logistic curve is fitted to the resulting points, that is, the 1000 different logistic curve generated is fitted for the prevalence data by varying the data before each fit with the ranges indicated next to the quality categories.

\section{RESULTS AND DISCUSSION}

The tools used for generating HIV estimates and the data used as mentioned above allows for production of estimated HIV prevalence and incidence trends from the beginning of the epidemic to the current year and projection for the future.

\section{National/States/Union Territory estimates of HIV adult prevalence}

While the adult HIV prevalence for each state and Union Territory was directly projected through EPP and Spectrum, the national adult HIV prevalence is determined through application of the simple aggregation number of PLHIV from all states divided by the total
Table 2 Adult HIV prevalence by sex and number of HIV infections for all ages with uncertainty bounds for the years 2008 and 2009, India

20082009

\begin{tabular}{|c|c|c|}
\hline \multicolumn{3}{|c|}{ Adult 15-49 HIV prevalence } \\
\hline Persons & $0.32 \%(0.26-0.41)$ & $0.31 \%(0.25-0.39)$ \\
\hline Female & $0.26 \%$ & $0.25 \%$ \\
\hline Male & $0.38 \%$ & $0.36 \%$ \\
\hline \multicolumn{3}{|c|}{ Number of HIV infections (all ages) } \\
\hline $\begin{array}{l}\text { Persons } \\
\text { (in million) }\end{array}$ & $2.44(1.97-3.09)$ & $2.40(1.93-3.04)$ \\
\hline \multicolumn{3}{|c|}{ Percentage distribution of HIV infection by sex } \\
\hline Female & 38.5 & 38.7 \\
\hline Male & 61.5 & 61.3 \\
\hline \multicolumn{3}{|c|}{ Percentage distribution of HIV infections by age group } \\
\hline$<15$ & 4.2 & 4.4 \\
\hline 15-49 & 83.3 & 82.4 \\
\hline $50+$ & 12.5 & 13.2 \\
\hline
\end{tabular}

adult population and calculated as a multiple of hundred to determine a percentage.

The adult HIV prevalence (males and females together) in India in 2008 is estimated as $0.32 \%$ with uncertainty bounds $0.26-0.41 \%$, and $0.31 \%$ in 2009 with uncertainty bounds $0.25-0.39 \%$. The adult HIV prevalence was estimated at $0.25 \%$ for women and $0.36 \%$ for men in 2009. It was estimated at $0.26 \%$ for women and $0.38 \%$ for men in 2008 (table 2). The results of 2008-2009 round of HIV estimates-in terms of trend and levels-are derived from a methodology that allows for readjustment of the prevalence curves on the basis of additional HSS data. Accordingly, the estimated HIV prevalence came out $0.36 \%$ for the year 2006 and $0.34 \%$ for the year 2007. As these are exactly the same value derived under the 2006 and 2007 round of HIV estimates, the consistency in results are indicative that the process adopted by the Working Group on Estimates and the results derived therein are valid and a sound base for analysis (figure 1).

\section{State-wise HIV adult prevalence}

States/Union Territory-wise estimates of adult HIV prevalence were the basis for estimating national adult

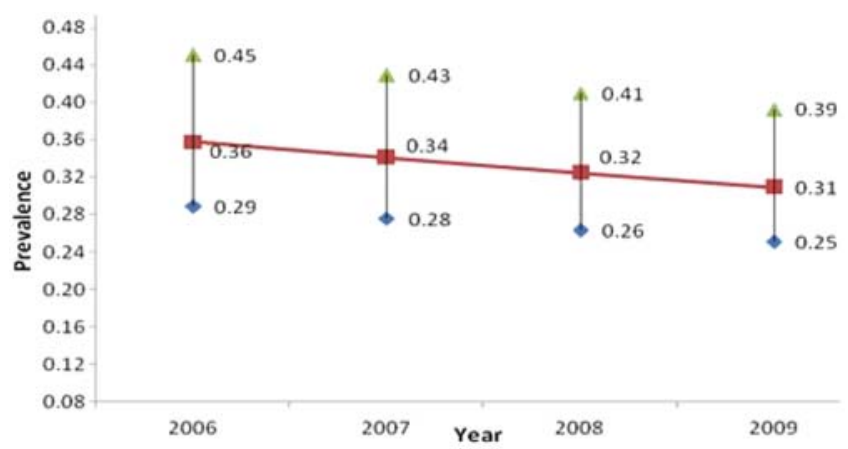

Figure 1 Adult HIV prevalence trend from 2006 to 2009, India. 
HIV prevalence as detailed in earlier sections of the report. Over and above this process, an uncertainty analysis was conducted independently for each state in Spectrum. The state-level estimates on adult HIV prevalence is provided in Map 1 (figure 2) and an analysis of central emerging trends is recapitulated therein.

As reflective from figure 3 , the HIV prevalence is on the decline in all states over the past 4-year period of 2006-2009; although the degree in decline varies slightly. For instance, the HIV prevalence trend appears stable in six high prevalence states, that is, Andhra Pradesh, Karnataka, Maharashtra, Manipur, Nagaland and Tamil Nadu and Mizoram (which is added to this group on the basis of consistently reporting HIV prevalence among ANC clinic attendees $>1 \%$ over the past 4 years in HSS). Further, in all three moderate prevalence states-Goa, Gujarat and Pondicherry, adult HIV prevalence trends is declining from 2006 to 2009.

Regarding the low-prevalence States/Union Territories, figure 3 highlights those where the trend for HIV prevalence is stable to increasing between 2006 and 2009. These include the seven States/Union Territories of
Arunachal Pradesh, Assam, Chandigarh, Jharkhand, Kerala, Meghalaya and Orissa.

The relatively greater increase in HIV prevalence is noted in Assam, Jharkhand and Orissa vis-à-vis Arunachal Pradesh, Kerala and Meghalaya. The estimated HIV prevalence for Chandigarh-as reflected in figure 3-is not considered a true reflection of the nature of the epidemic in the Union Territory. While noting the increase in the estimated HIV prevalence, it must be considered that the trend is attributable to the services Chandigarh provides to PLHIV from the neighbouring states of Punjab, Haryana and even Himachal Pradesh. As ART information is included as one of the parameters for the projection, the resulted trend is showing an increase, independent of the trend observed in HSS. A separate analysis of the HIV epidemic in Chandigarh is thus required to take into consideration the evidence and the trends of neighbouring states.

Figure 3 highlights the low prevalence states of Delhi, Haryana, Punjab, Rajasthan, Uttar Pradesh and West Bengal where HIV prevalence is either stable or declining. Evidently, the degree for decline in HIV prevalence

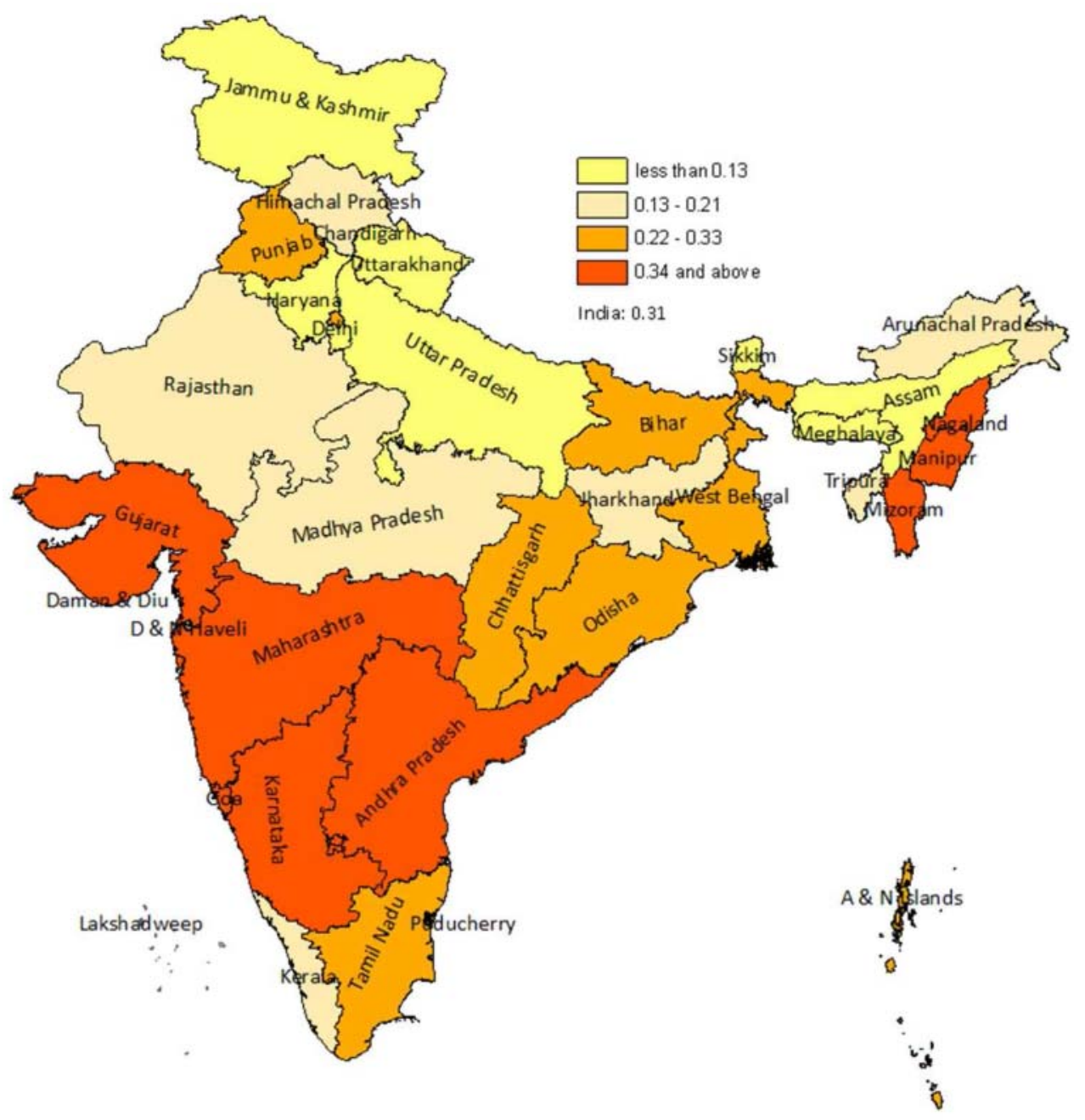

Figure 2 Map 1: Adult HIV Prevalence (\%) by state, 2009. 

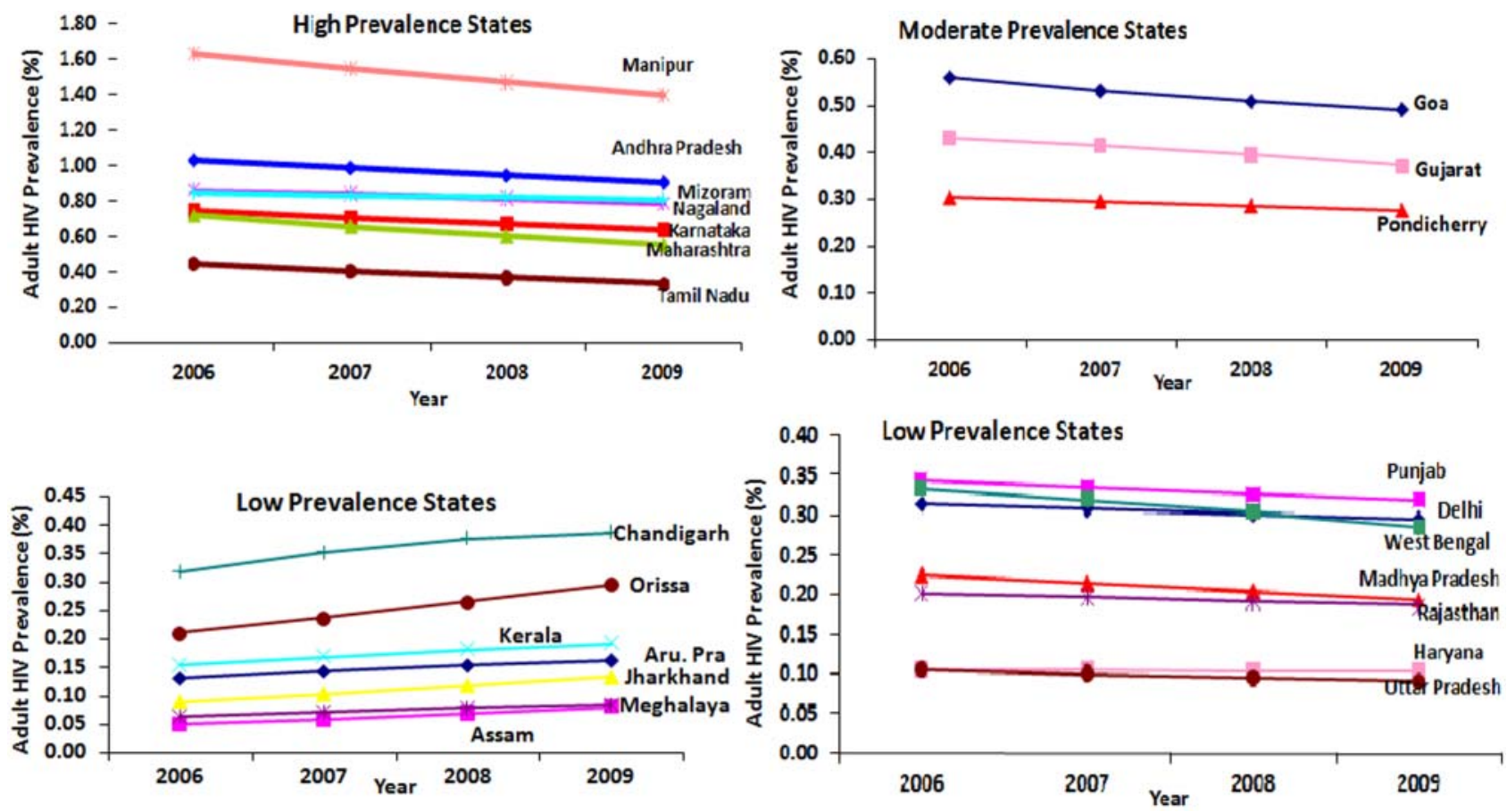

Figure 3 Adult HIV prevalence trend from 2006 to 2009, Indian states.

is not uniform between these states which may be on account of the programmatic impact of ART coverage, and the strength of the prevention interventions, etc. The decline in HIV prevalence is lower in Haryana and Punjab vis-à-vis Delhi, Rajasthan, Uttar Pradesh and West Bengal.

\section{National and State estimates of number of PLHIV}

In 2009, the total number of PLHIV in India was estimated at 2.4 million (uncertainty bounds of 1.93-3.04 million) whereas in 2008, it was 2.44 million people who were living with HIV within the uncertainty bounds of 1.97-3.09 millions. Among PLHIV, by sex approximately $61 \%$ are male and $39 \%$ are female and by age the percentage distribution of HIV infection is estimated at $4 \%$ are children below the age of 15 years, $83 \%$ are adults aged $15-49$ years and the rest $13 \%$ are over 50 years of age (table 2).

The four high-prevalence states of South India account for $57 \%$ of all HIV infections in the country. While Andhra Pradesh accounts for 500000 cases; Maharashtra accounts for 420000 cases, Karnataka accounts for 250000 cases and Tamil Nadu accounts for 150000 cases. Over 100000 PLHIVs are reported in West Bengal, Gujarat, Bihar and Uttar Pradesh and together these states account for $22 \%$ of HIV infections in India. The number of PLHIVs in Punjab, Orissa, Rajasthan and Madhya Pradesh range from 50000 to 100000 and these states collectively account for $12 \%$ of HIV infections. Thus, while the states noted above are with low HIV prevalence; a large number of PLHIVs are reported due to the states' overall large population size (Map 2; figure 4).
The percentage distribution of HIV burden among high-prevalence states vis-à-vis the remaining states in India is $57 \%$ and $43 \%$, respectively. Among the highprevalence states, Andhra Pradesh accounts for the greatest proportion of cases at $21 \%$ vis-à-vis the other states. Following Andhra Pradesh, Maharashtra accounts for approximately $18 \%$ of HIV infection, Karnataka and Tamil Nadu reportedly account for $10 \%$ and $7 \%$ of all cases, whereas Manipur and Nagaland account for $1 \%$ of the estimated total.

\section{CONCLUSIONS}

The India HIV estimates 2008-2009 indicate a slowdown in the AIDS epidemic. National adult HIV prevalence, or the number of adults living with HIV as a proportion of the total population, has declined by $0.05 \%$ points from $0.36 \%(0.29-0.45 \%)$ in 2006 to $0.31 \%$ (0.25$0.39 \%$ ) in 2009. Though the estimation of 2008-2009 has utilised EPP and Spectrum tool against UNAIDS/ WHO workbook and Spectrum in 2006, the results of 2008-2009 round of estimates match perfectly with previously announced estimates. However, the results of 2008-2009 round of estimates cannot be directly compared with the previous years because the methodology and data used to produce the estimates have gradually changed as a result of ongoing enhancement of knowledge on the epidemic.

Adult HIV prevalence is either stable or declining in the high-prevalence states whereas the trend is varying across the low-to-moderate prevalence states. Among the high-prevalence states, the HIV prevalence has declined in Tamil Nadu between 2006 and 2009 to reach levels of 


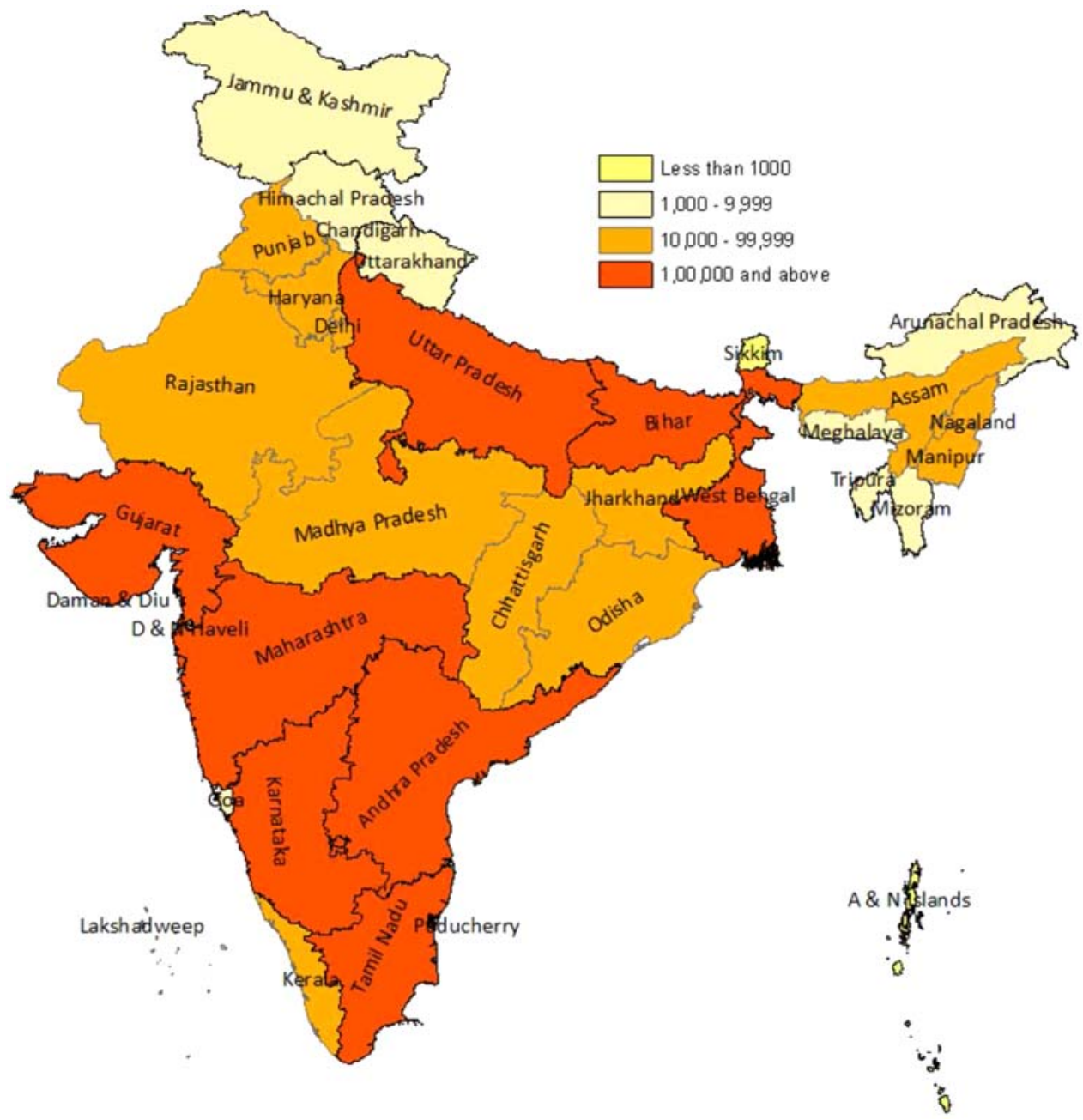

Figure 4 Map 2: Estimated number of HIV infection by state, 2009.

$0.37 \%$ in 2008 and $0.33 \%$ in 2009. Manipur shows a declining trend over the past 4 years. Andhra Pradesh, Karnataka, Maharashtra and Nagaland show either a plateau or a slightly declining trend over the time period 2006-2009.

In the low-prevalence states of Chandigarh, Orissa, Kerala, Jharkhand, Uttarakhand, Jammu and Kashmir, Arunachal Pradesh and Meghalaya; adult HIV prevalence has risen over the last 4 years which warrants the need for strengthening the trend of the HIV epidemic.

In descending order, states with the highest adult HIV prevalence in 2009 included Manipur (1.4\%); followed by Andhra Pradesh $(0.90 \%)$, Mizoram $(0.81 \%)$, Nagaland $(0.78 \%)$, Karnataka $(0.63 \%)$ and Maharashtra $(0.55 \%)$. Besides these, the states of Goa, Chandigarh, Gujarat, Punjab and Tamil Nadu have an estimated adult HIV prevalence greater than national prevalence $(0.31 \%)$. Delhi, Orissa, West Bengal, Chhattisgarh and Pondicherry have an estimated adult HIV prevalence of $0.28-0.30 \%$. Other states have lower levels of HIV.

The total number of PLHIV in India is estimated at 2.4 million (uncertainty bounds of 1.93-3.04 million) in 2009. Children under 15 years of age account for $4.4 \%$ of all infections, while people aged 15-49 years account for $82.4 \%$ of all infections. Thirty-nine per cent of all HIV infections are estimated to be among women. This amounts to 0.93 million women with HIV in India.

\section{Author affiliations}

${ }^{1}$ National Institute of Medical Statistics, ICMR, New Delhi, India

${ }^{2}$ India Country Office, UNAIDS, New Delhi, India

${ }^{3}$ WHO India Office, New Delhi, India

${ }^{4}$ National AIDS Control Organization, New Delhi, India

${ }^{5}$ Centre of Community Medicine, All India Institute of Medical Sciences, New Delhi, India

${ }^{6}$ National Institute of Health and Family Welfare, New Delhi, India

Acknowledgements We are thankful to experts of Technical Resource Group (TRG) of HIV Surveillance and Estimation of India at National AIDS Control Organization (NACO), Department of AIDS Control, Ministry of Health and Family Welfare, Government of India, Delhi.

Contributors The authors are the members of the working group on India's HIV estimation and have contributed equally in the design, analysis, interpretation and critical review of the paper.

Competing interests None.

Provenance and peer review Not commissioned; externally peer reviewed. Data sharing statement No additional data are available. 


\section{REFERENCES}

1. National Institute of Health \& Family Welfare. Country Report on Annual HIV Sentinel Surveillance, 2006, New Delhi: National AIDS Control Organization (NACO), Ministry of Health \& Family Welfare, 2007.

2. International Institute for Population Sciences (IIPS) and Macro International. India, IIPS, Mumbai: National Family Health Survey, 2005-2006 (NFHS-3), 2007.

3. Dandona L, Lakshmi V, Sudha T, et al. A population based study of human immunodeficiency virus in south India reveals major differences from sentinel surveillance-based estimates. BMC Med 2006;4:31-49.

4. Dandona L, Lakshmi V, Kumar GA, et al. Is the HIV burden in India being over estimated? BMC Public Health 2006;6:308-17.

5. Pandey A, Reddy DCS, Ghys $P$, et al. Improved estimate of India's HIV burden in 2006. Indian J Med Res 2009;129:50-8.

6. Pandey A, Reddy DCS, Thomas M. What lies behind the fall in the HIV population in India? Eco Polit Wkly December 2008:27:15-17.

7. Lyerla R, Gouws E, Garcia-Calleja JM, et al. The 2005 Workbook: an improved tool for estimating HIV prevalence in countries with low level and concentrated epidemics. Sex Transm Infect 2006; 82(Suppl III):iii41-4

8. Stover J, Walker N, Grassly NC, et al. Projecting the demographic impact of AIDS and the number of people in need of treatment: updates to the Spectrum projection package. Sex Transm Infect 2006;82(Suppl III):iii45-50.

9. Pandey A, Reddy DCS, Thomas M, et al. Prevalence and burden of HIV in India 2007-an update of estimates, Demogr India 2010;39:239-45.

10. UNAIDS Global Reference group on Estimates, Modelling and Projections http://www.epidem.org (accessed 15 December 2011).

11. UNAIDS. 2010, http://www.unaids.org/en/dataanalysis/tools/ estimation projection package epp/
12. Stover J, Johnson $\mathrm{P}$, Zaba B, et al. The Spectrum projection package: improvements in estimating mortality, ART needs, PMTCT impact and uncertainty bounds. Sex Transm Infect 2008;84:i24-30.

13. National AIDS Control Organization (NACO). National AIDS Control Programme-III (NACP-III). New Delhi: NACO Ministry of Health and Family Welfare, New Delhi, 2006.

14. National AIDS Control Organization. Technical Report India HIV Estimates 2008-2009 National Institute of Medical Statistics, ICMR. India: Ministry of Health \& Family Welfare, 2010.

15. Sample Registration System, Statistical reports, Office of the Registrar General India, New Delhi, 2010.

16. Census of India 1981. New Delhi: Office of the Registrar General India.

17. Census of India 1991. New Delhi: Office of the Registrar General India.

18. Census of India 2001. New Delhi: Office of the Registrar General India.

19. Population Projections for India and States 2001-2026, Report of the Technical Group on Population Projections. New Delhi: National Commission on Population, Ministry of Health \& Family Welfare, May 2006.

20. Behavioural Surveillance Survey, 2006-Female sex Workers (FSW) \& their clients. New Delhi: National AIDS Control Organization (NACO), Ministry of Health \& Family Welfare.

21. National AIDS Control Organization, National AIDS Control Programme CMS. India: Ministry of Health \& Family Welfare.

22. Bachani D, Sogarwal R, Rao KS. A population based survey on HIV prevention in Nagaland, India. SAARC J Tuberculosis, Lung Dis HIV/AIDS 2009;6:1-11.

23. Stover J, Johnson $\mathrm{P}$, Hallett $\mathrm{T}$, et al. The Spectrum projection package: improvements in estimating incidence by age and sex, mother-to-child transmission, HIV progression in children and double orphans. Sex Transm Infect 2010;86:ii16-21.

24. Grassly NC, Morgan M, Walker N, et al. Uncertainty in estimates of HIV/AIDS: the estimation and application of plausibility bounds. Sex Transm Inf 2004;80:31-8. 